\title{
Anabases
}

ANABASES Traditions et réceptions de l'Antiquité

19 | 2014

Varia

\section{Une Économie antique en version française}

\section{Christophe Pébarthe}

\section{(2) OpenEdition}

Journals

Édition électronique

URL : http://journals.openedition.org/anabases/4562

DOI : 10.4000/anabases.4562

ISSN : 2256-9421

\section{Éditeur}

E.R.A.S.M.E.

\section{Édition imprimée}

Date de publication : 1 avril 2014

Pagination : 55-68

ISSN : 1774-4296

\section{Référence électronique}

Christophe Pébarthe, "Une Économie antique en version française », Anabases [En ligne], 19 | 2014, mis en ligne le 01 avril 2017, consulté le 20 octobre 2019. URL : http://journals.openedition.org/anabases/ 4562 ; DOl : 10.4000/anabases.4562 
Anabases 19 (2014), p. 55-68

\section{Une Économie antique en version française}

Christophe Pébarthe

« Est-ce à dire que je vous propose une conférence "sur" Proust ? Oui et non. Ce sera, si vous le voulez bien : Proust et moi. Quelle prétention ! [...] en disposant sur une même ligne Proust et moi-même, je ne signifie nullement que je me compare à ce grand écrivain, mais d'une manière tout à fait différente, que je m’identifie à lui [...] je ne m'identifie pas à l'auteur prestigieux d'une œuvre monumentale, mais à l'ouvrier, tantôt tourmenté, tantôt exalté, de toute manière modeste, qui a voulu entreprendre une tâche à laquelle, dès l'origine de son projet, il a conféré un caractère absolu ${ }^{1}$. " Ainsi Roland Barthes s'exprimait-il lors d'une conférence au Collège de France en 1978, tel pourrais-je m'exprimer, substituant Finley à Proust, ressentant la prétention et affichant le choix délibéré de chercher "l'ouvrier " derrière l'historien, s'identifiant, par la pratique historienne et non par la valeur, à l'auteur de l'Économie antique. « Ce sera [donc], si vous le voulez bien : [Finley] et moi. " Ce moi haïssable ne saurait en effet être dissimulé. À certains moments - et peut-être même encore aujourd'hui -, université Bordeaux 3, élève d'Alain Bresson, valaient brevet d'anti-finleyisme primaire. Quelle que fût la réalité de cette hostilité à l'encontre de l'Économie antique, il semble nécessaire d'interroger l'éventuelle singularité de la lecture bordelaise de Finley, avant de rendre compte de la réception qu'a connue L'économie antique en France, en soulignant - et en cherchant à expliquer - l'apparent paradoxe d'une version française possiblement antérieure à The Ancient Economy, Économie et sociétés en Grèce ancienne par Michel Austin et Pierre Vidal-Naquet.

1 R. BARThes, Essais critiques IV. Le bruissement de la langue, Paris, 1984, p. 313-314. 


\section{Comment fut lu The Ancient Economy depuis Bordeaux}

La relation que les historiens bordelais entretiennent avec The Ancient Economy commence en 1975, dans la Revue des Études Anciennes. Si l'édition originale n'avait pas donné lieu à compte rendu, la traduction française, due à Max P. Higgs, est recensée par Raymond Descat ${ }^{2}$. Ce dernier, alors assistant d'Histoire ancienne à Bordeaux, était en train de préparer une thèse d'État sur le travail, sous la direction de Pierre Lévêque, tous deux réunis par un même intérêt pour le marxisme. Cette position explique sans doute la prudente conclusion : "Dans une discipline où les questions sont plus nombreuses que les réponses, ce livre suscite la réflexion et l'oriente souvent dans des directions nouvelles, ce qui n'est pas le moindre signe de sa valeur ${ }^{3}$. " Mais ce compte rendu fait apparaître des points saillants de l'œuvre. Après avoir défini son objet précis, non pas "une histoire de l'économie antique " mais "une étude du comportement économique des Anciens ", il précise la finalité recherchée par Finley : "Ne cherchons donc pas des définitions précises au plan de la chronologie ou de la géographie, comme on en attendrait de l'étude d'un mode de production. Finley dégage plutôt une sorte d'idéal-type à la Weber - l'homme de l'Antiquité. " Il insiste également sur " cette interrogation sur la rationalité économique de l'homme antique qui fait le grand intérêt du livre ». Néanmoins, il laisse apparaitre les difficultés d'une altérité des sociétés anciennes définies simplement par l'absence du marché et ses conséquences, et prend ses distances aussi bien avec Finley qu'avec Polanyi : «Le lecteur [...] se demande souvent s'il faut s'en tenir à l'idée que l'économie n'existe pas en tant que catégorie intellectuelle ou bien admettre qu'il est “inextricablement” lié, par la structure même de l'économie, à d'autres facteurs (politique, religieux...), ce qui n'est pas la même chose. »

De même, Raymond Descat souligne qu' " il n'y a pas opposition entre une conduite économique et une conduite qui ne l'est pas puisque toutes deux concernent des biens matériels. Elles sont différentes pour des faits qui tiennent à la formation sociale tout entière. Le psychologisme masque notre ignorance ». Sans doute en raison de sa proximité intellectuelle avec le marxisme, il insiste sur la dimension matérielle. Si le risque a toute sa place "dans la mentalité grecque classique ", il porte non pas sur la croissance mais sur « la conservation du niveau matériel ». Raymond Descat se réclame alors de l'anthropologie la plus fonctionnaliste, expliquant la reproduction humaine par un calcul entre ce qui a été donné à l'enfant et ce que ce dernier donnera à l'âge adulte ; et de conclure : «Le discours d'Ischomaque a sur ce plan aussi sa parfaite rationalité. "La distance par rapport à Finley est ici nette, puisqu'il ne s'agit pas tant de mettre en évidence l'absence de rationalité en Grèce ancienne que de délimiter les contours d'une rationalité grecque, sous conditions matérielles particulières. Elle n'est toutefois pas radicale, une différence de degré plutôt que de nature, puisque l'historien

2 REA 78-79 (1976-1977), p. 337-339.

3 Ibid., p. 339. 
de Cambridge affirme dans le premier chapitre : "Nous devons, je crois, rechercher d'autres concepts et d'autres modèles qui conviennent à l'économie antique, et non (du moins pas nécessairement) à la nôtre ${ }^{4}$. " En l'occurrence, le modèle commun est à chercher dans l'anthropologie et repose sur une même conception de l'altérité grecque.

En 1987, dans la même revue, Raymond Descat consacre un article à l'économie athénienne au IV siècle ${ }^{5}$. Il affirme d'emblée l'accord autour du cadre dessiné par L'économie antique. Toutefois, dans la conclusion, un écart apparaît. L'économie athénienne est qualifiée d' "économie partiellement marchande où le marché conquiert une partie des circuits économiques, mais pas tout, et où il n'y a pas véritablement de marché du travail ${ }^{6}$ ". Les termes sont mesurés mais le débat est orienté autour de la question du marché, un concept que Finley n'a pas défini dans The Ancient Economy, sinon pour proclamer l'absence d'un grand marché unifié à l'échelle de la Méditerranée ${ }^{7}$. Sans avoir donc l'impression de rompre véritablement avec ce modèle, Raymond Descat peut considérer qu'Athènes est entrée dans l'économie de marché au V siècle. Son collègue bordelais, Alain Bresson, intervient dans le même volume de la Revue des études anciennes avec son "Aristote et le commerce extérieur ${ }^{8}$ ". La distance avec L'économie antique est ici beaucoup plus nette, même si la pertinence de la critique finleyienne du modernisme est soulignée dès l'abord ${ }^{9}$. Exprimant une méfiance à l'encontre de toute généralisation précoce, ayant pour seule finalité de se substituer à la nouvelle orthodoxie, Alain Bresson en appelle à une discussion raisonnée du modèle. Dans cet article, il se consacre au supposé désintérêt de la cité grecque pour le commerce, sinon pour assurer la trophè. Il montre d'abord que l'autarkeia aristotélicienne ne désigne pas l'autarcie, autrement dit l'autosuffisance, mais l'équilibre entre les importations et les exportations. D'autres auteurs anciens sont convoqués, ce qui permet de faire apparaître que cette conception est partagée et non seulement aristotélicienne. Puis, l'épigraphiste Alain Bresson, formé par Jean Pouilloux et Louis Robert, fait intervenir les inscriptions pour asseoir sa démonstration, sans négliger les sources littéraires, et non sans avoir auparavant montré à partir d'un passage d'Aristote que les cités concluaient des accords (synthékai et symbolai) sur les exportations et les importations ${ }^{10}$. Ainsi, « la trophè était certes un souci essentiel mais ce sujet ne pouvait pas à lui seul tenir lieu de politique de la cité en matière d'échanges extérieurs. En effet, pour pouvoir importer, il fallait aussi exporter et s'en donner les moyens. [...] Il suffira ici de constater du moins que, en tant qu'États, les cités ne pouvaient se

4 M.I. Finley, L'économie antique, Paris, 1975, p. 28-29.

5 "L'économie d'une cité grecque au IV siècle avant J.-C. : l'exemple athénien ", REA 89 (1987), p. 239-252.

$6 \quad$ Ibid., p. 252.

7 FinLEY, L'économie antique, p. 22 et 38.

8 A. BRESSON, "Aristote et le commerce extérieur ", REA 89 (1987), p. 217-238.

$9 \quad$ Ibid., p. 218.

10 Arstt., Rhet., 1360a avec A. BressON, « Aristote ", p. 227-228. 
désintéresser du commerce extérieur ${ }^{11}$ ». La volonté est alors clairement de rompre avec la nouvelle orthodoxie, non d'en aménager certains aspects.

Il n'est sans doute pas fortuit de constater que quelques années plus tard Raymond Descat prend à son tour position sur " le rôle de l'État dans le commerce en Grèce ancienne $^{12}$ ». La logique de champ, en l'occurrence ici l'histoire grecque bordelaise, semble jouer à plein. Il reprend implicitement le raisonnement d'Alain Bresson, par le biais de l'historiographie et la discussion des thèses de Johannes Hasebroek. Tout en exprimant son plein accord sur l'autarkeia, il considère " qu'une fois cette évidence rappelée, le problème de fond n'est pas réglé pour autant. Certes, l'exportation est bien une réalité, mais cela ne dit rien sur la politique de la cité dans les échanges ${ }^{13}$ ". C'est ce dernier point que Raymond Descat cherche à préciser dans cet article. Pour lui, "Aristote critique donc une vision prosodique et fiscale de la politique de la cité ${ }^{14}$ ". L'intervention de la cité cherche à corriger des dysfonctionnements consécutifs aux actions des riches producteurs mais surtout à " organiser [...] un espace commun de circulation des biens le plus large possible ${ }^{15}$ ». La cité entend donc attirer des importations, veille à ce que les productions locales s'échangent et évite que la monnaie soit rare et s'échappe du territoire pour que son usage se développe. "À la différence des États modernes, et pour des raisons inhérentes à son histoire, il n'y a pas dans la cité grecque de liens conceptuels (et donc de politique consciente) entre cette intervention dans la circulation et les autres parties de l'économie qui restent pour l'essentiel du domaine individuel ${ }^{16}$. " De ce point de vue, l'agora est un marché, c'est-à-dire un lieu concret d'échanges ${ }^{17}$. Désormais, la question des échanges est devenue essentielle, alors même qu'elle n'occupe pas une place particulière dans The Ancient Economy.

L'année suivante, Raymond Descat discute le modèle finleyien dans sa globalité. Il souligne que ce dernier s'est imposé en donnant une explication politique du début du monde antique. "Ce qui se passe à l'époque classique est le triomphe de la cité comme institution et la construction par la cité d'une domination culturelle sur l'économie. [...] Cette idée s'est d'autant plus facilement imposée qu'elle est en accord avec une conception dominante dans les études grecques qui fait de l'installation de la structure politique de la cité un processus cognitif clair ${ }^{18}$. " La référence aux Origines

11 A. BRESSON, "Aristote ", p. 238.

12 "La cité grecque et les échanges. Un retour à Hasebroek", in J. Andreau, P. Briant \& R. Descat (éd.), Les échanges dans l'Antiquité : le rôle de l'État. Entretiens d'archéologie et d'histoire, Saint-Bertrand-de-Comminges, 1994, p. 11-30.

13 Ibid., p. 15.

14 Ibid., p. 18.

15 Ibid., p. 19.

16 Ibid., p. 27.

17 Sur ce point, cf. R. DESCAT, " La loi de Solon sur l'interdiction d'exporter les produits attiques ", in A. Bresson \& P. Rouillard (éd.), L'emporion, Paris, 1993, p. 145-161.

18 R. DESCAT, "L'économie antique, un modèle en question ", AHSS 50 (1995), p. 963. 
de la pensée grecque de Vernant est transparente. Raymond Descat pose alors la question de l'application généralisée d'un tel modèle à l'ensemble du monde grec, autre façon de s'interroger sur le caractère paradigmatique ou non de l'économie athénienne. Il montre à tout le moins que cette dernière n'était en rien une exception, mais pour marquer une certaine distance avec le modèle finleyien. Au cours de la période classique, le nombre des grandes cités a eu tendance à s'accroître, ce qui vaut généralisation de la stratégie d'autarkeia ${ }^{19}$. Par ailleurs, Raymond Descat rejette toute opposition entre agriculture et marché. Il existe au contraire une relation étroite entre les deux, " un aspect de l'utilisation des surplus, une occasion dont il faut savoir profiter ${ }^{20}$ ». Bien plus, ajoute-t-il, dans un renversement complet du modèle finleyien, "le marché s'inscrit dans une logique de conservation de l'oikos ${ }^{21}$ ". Lorsque la cité intervient, c'est pour maintenir l'équilibre, pour que les biens sur l'agora, considérés comme koina, circulent, ce qui implique une politique d'importation et une politique d'exportation, ainsi qu'une action sur la production. Dès lors, la monnaie et le crédit sont décisifs puisqu'ils permettent la circulation, notamment entre agriculture et activités non agricoles. In fine, tout en affirmant la victoire du modèle finleyien, Raymond Descat s'en écarte fortement. L'heure est à la recherche d'un nouveau modèle.

Dans un article paru dans la revue Ktêma, Alain Bresson en jette les bases en marquant un écart sensible avec Raymond Descat. La question de la cité est devenue centrale. La polis n'est pas un État et gère donc ses ressources comme un oikos, c'est-àdire qu' elle cherche à accroître ses ressources, ses prosodoi. "Au point de départ, l'État apparaît donc davantage comme un organe de répartition des profits faits collectivement que dans un rôle d'autorité abstraite ou une fonction de régulation et de législation $^{22}$." Dans cette optique, la guerre, et le partage du butin qu'elle implique en cas de victoire, est un moyen de profit. La citoyenneté est pensée comme une " participation à un club distribuant des dividendes ${ }^{23}$ ». Outre les guerres dont on attend des prosodoi, les cités disposent d'autres projets pour que leurs revenus profitent à leurs citoyens, comme les constructions publiques. "Dans la paix comme dans la guerre, l'État restait donc avant tout une machine à collecter et à redistribuer les prosodoi ${ }^{24}$. » Tout en rejetant Finley donc, Alain Bresson en appelle à la construction d'une réflexion reposant sur les catégories conceptuelles des Grecs de l'Antiquité, ce que The Ancient Economy avait annoncé sans le faire. Sans s'interdire des parallèles avec la période contemporaine, dans le chapitre inédit et conclusif de la Cité marchande, Alain Bresson poursuit la démarche entamée dans Ktêma et souligne les spécificités de l'économie grecque.

19 Ibid., p. 965 et n. 16 reprend la définition proposée par Alain Bresson, sans toutefois y faire référence.

20 Ibid., p. 968.

21 Ibid.

22 A. Bresson, La cité marchande, Bordeaux, 2000, p. 248.

23 Ibid., p. 250.

24 Ibid., p. 252. 
"La distinction du système libéral contemporain entre le public, i. e. l'État, et le privé, i. e. l'économie, n'existait pas dans le monde de la cité grecque antique. C'est en fait la catégorie des prosodoi qui est décisive pour pouvoir analyser ces sociétés ${ }^{25}$. "Cette différence étant posée, il devient possible de s'interroger sur l'existence de structures marchandes antiques, sans revenir au modernisme le plus strict. C'est ainsi qu'il faut entendre la conclusion d'Alain Bresson. "L'économie de la Grèce des cités relève donc pleinement de la catégorie des économies à marché, même s’il s'agissait d'un marché qui fonctionnait différemment du marché contemporain (c'est un truisme que de le dire) ", tout en précisant un peu plus loin que " cela ne signifie nullement que l'on doive réduire l'économie de la Grèce des cités au marché ${ }^{26}$ ».

C'est désormais autour du marché que la discussion bordelaise va s'organiser, dans deux directions différentes, à certains égards opposées. Dans une série d'articles, Raymond Descat prolonge la réflexion ouverte en 1993 dans son étude de la loi de Solon ${ }^{27}$. Marquant une distance avec toute entreprise de conceptualisation, il entend décrire l'agora comme un marché concret, précisément pour sortir de la nasse que représente le débat entre primitivisme et modernisme. Comme il l'écrit dans la Revue de Synthèse, "le marché antique [...] est plutôt le signe de l'appartenance à un camp, utilisé de manière polémique, qui se substitue à une réflexion sur le concept luimême $^{28}$ ". Il assimile le marché antique à l'agora, le concept et l'institution d'un côté et la réalité historique, et même archéologique plus récemment, de l'autre. Au contraire, Alain Bresson propose les étapes d'une démarche conceptuelle visant à élaborer une nouvelle description $\mathrm{du}$ fonctionnement $\mathrm{du}$ marché antique, inscrit dans un cadre théorique spécifique, le néo-institutionnalisme, selon lequel toute économie possède des institutions dont le fonctionnement vise à lever les incertitudes dans un contexte de rareté2 ${ }^{29}$. Les travaux de Douglass North constituent de ce point de vue un point de départ indispensable. Ce programme trouve son achèvement dans les deux volumes parus en 2007 et 2008 chez Armand Colin, L'économie de la Grèce des cités. Par le recours au néo-institutionnalisme, très en vogue aux États-Unis, L'économie antique en version bordelaise pouvait alors faire retour au pays natal de Moses Finley.

25 Ibid., p. 271.

26 Ibid., p. 304. et 306

27 R. DESCAT, "Loi de Solon ", et ID, "Remarques sur la naissance du marché en Grèce archaïque ", in Ph. Clancier, Fr. Joannès, P. Rouillard \& A. Tenu (éd.), Autour de Polanyi. Vocabulaire, théories et modalités des échanges. Nanterre 12-14 juin 2004, Paris, 2005, p. 239-247.

28 R. DesCat, "Le marché dans l'économie de la Grèce antique ", Revue de Synthèse 127 (2006), p. 257.

29 A. BRESSON, «Économie et institution. Bilan critique des thèses polanyiennes et propositions nouvelles ", in Clancier et al., (éd.), Autour de Polanyi, p. 97-111. 
On l'aura compris, la démarche entreprise ici consiste à " comprendre, [c'est-àdire] comprendre d'abord le champ avec lequel et contre lequel on s'est fait ${ }^{30}$ ". Il est donc décisif de décrire l'état du champ au moment où tel y entre. À cet égard, il n'est pas question d'une énième mise en scène de l'exercice de l'ego-histoire, mais bien de lire des trajectoires pour mieux comprendre des pensées, des savoirs produits. Cette lecture "bordelaise » de Finley a, semble-t-il, orienté la réception française de l'Économie antique, c'est-à-dire la discussion des thèses défendues dans cet ouvrage autour de notions économiques. D’autres débats auraient pu pourtant naître, à partir de l'ancrage français des thèses finleyiennes.

\section{The Ancient Economy, une co-production franco-finleyienne?}

Au moment de la parution de la traduction française de The Ancient Economy, les travaux de Moses Finley sont déjà bien connus en France. Dès 1953, Paul Cloché avait recensé Studies in Land and Credit in Ancient Athens, 500-200 BC. The HorosInscriptions dans la Revue historique et dans la Revue belge de philologie et d'histoire ${ }^{31}$. À cette date, ce professeur à la retraite, âgé de 72 ans, qui a accompli toute sa carrière à l'université de Besançon, est un universitaire reconnu. Toutefois, il n'est pas indifférent de remarquer sa position : il est agrégé d'histoire et de géographie, il n'est donc pas philologue, et provincial ${ }^{32}$. Dans ces deux recensions, Paul Cloché s'en tient à un résumé faisant apparaître quelques points saillants : la nature foncière des patrimoines athéniens ; le recours aux accords verbaux dominant dans le domaine des hypothèques ; l'endettement en vue de payer des impôts ou de doter une fille et non pour accroître les ressources. Il conclut en saluant « un savant ouvrage qui rendra maints services aux recherches des historiens et des juristes ${ }^{33}$ ». Ce recueil d'inscriptions n'a évidemment pas échappé à l'attention de Jeanne et Louis Robert qui lui consacrent une courte note dans le Bulletin épigraphique de $1953^{34}$. Aucune des thèses fortes de l'ouvrage n'est rappelée. Pour l'essentiel, ils se contentent de quelques remarques formelles et d'une affirmation prudente : «On sera sans doute amené à faire des objections à certaines interprétations et à repousser certains raisonnements. " Ainsi, en 1953, en France, la thèse publiée de Moses Finley n'est pas véritablement lue comme un ouvrage d'histoire

30 P. Bourdieu, Esquisse pour une auto-analyse, Paris, 2004, p. 15.

31 Revue historique 210 (1953), p. 342-343.

32 Cf. P. Chantraine, "Éloge funèbre de M. Paul Cloché, correspondant à l'Académie ", CRAI (1961), p. 358-359.

33 RBPH 31 (1953), p. 619-622.

34 J. \& L. Robert, "Bulletin épigraphique », REG 66 (1953), p. 124-125 n. 23. 
économique, encore moins comme une prise de position dans la querelle entre primitivistes et modernistes ${ }^{35}$.

L'année suivante, dans un aperçu des trois derniers quarts de siècle de recherches sur l'économie grecque, Édouard Will ne fait pas référence à Studies in Land and Credit $^{36}$. Il ne mentionne que l'article de 1935 consacré à l'étude de la terminologie grecque du commerce. Deux raisons peuvent expliquer cette absence. La première porte sur la situation d'Édouard Will. Achevant sa thèse d'État sur Corinthe à l'époque archaïque, sans doute n'avait-il pas eu le temps nécessaire pour prendre acte de la prise de position finleyienne dans le débat sur la nature de l'économie antique. La deuxième procède de la finalité poursuivie. Comme il l'indique lui-même, ce rappel historiographique vise à porter à la connaissance des historiens français une querelle qui, pour l'essentiel, s'est tenue en allemand. Ce faisant, il expose malgré tout sa propre conception de l'économie grecque. S'il marque ses distances avec les thèses opposées de Bücher et de Meyer présentées comme " des théories abstraites, conçues avant qu'un patient travail d'analyse eût été effectué sur les Realien ", il n'en affirme pas moins la validité de la " critique pertinente des exagérations modernistes ${ }^{37}$ ". Il ébauche aussi une méthode à suivre pour dépasser la querelle. Aux abstractions de la science économique, il faut opposer le concret, au progrès technique et à l'évolution, la technique statique. Afin de se placer dans " un nouvel état d'esprit plus objectif ", il est nécessaire selon Will de fournir un effort d'ordre psychologique : "La mentalité économique des Grecs anciens n'était, de toute évidence, pas la même que la nôtre. Il convenait donc de nous dépouiller de notre propre mentalité d'hommes du XIX ${ }^{\mathrm{e}}$ ou du XX $\mathrm{XX}^{\mathrm{e}}$ siècle, pour chercher à pénétrer la psychologie de l'homo xeconomicus antique ${ }^{38}$. "Enfin, il soulignait avec force l'importance des livres de Johannes Hasebroek, en particulier Staat und Handel im alten Griechenland (Tubingen, 1928), dans lesquels il retrouvait la critique du modernisme et lisait surtout l'importance de la cité. Il concluait sur la nature essentiellement agricole de l'économie grecque et son corollaire " une production artisanale archaïque "; un commerce extérieur n'ayant pour seule finalité que la trophè " déterminant chez une catégorie indispensable d'individus une tendance à l'extraversion cosmopolite antagoniste de la tendance autarcique fondamentale "; un volume des échanges faible ; un fort risque dans les activités commerciales à l'origine du développement de la chrématistique ${ }^{39}$. C'est avec ce programme en tête que, quelques

35 Tout en saluant une " contribution de grande valeur " et en discutant en détail certaines analyses de Finley dans son compte rendu de 1953, L. Gernet n'inscrit pas non plus cet ouvrage dans la querelle (cf. Les Grecs sans miracle. Textes 1909-1960, Paris, 1983, p. 121-129).

36 "Trois quarts de siècle de recherches sur l'économie grecque antique ", AESC 9 (1954), p. 7-22.

37 Ibid., p. 11-12.

38 Ibid., p. 12.

39 Ibid., p. 19. 
années plus tard, Édouard Will confie à Moses Finley le rapport sur la Grèce classique lors de la Deuxième conférence internationale d'histoire économique qui se tint à Aix en 1962.

Mais la rencontre décisive est celle de Pierre Vidal-Naquet avec Moses Finley ${ }^{40}$. Agrégé d'histoire en 1955, assistant à l'université de Caen puis à Lille, détaché au CNRS en 1962, Vidal connaît alors les deux années " parmi les plus productives de [sa] vie ", au cours desquelles il suit les séminaires de Jean-Pierre Vernant, d'André Aymard, de Roger Rémondon qui lui présente Claire Préaux et de Louis Robert ${ }^{41}$. Parmi les questions qui l'intéressent alors, il y a celle de l'historicité de la société homérique. À cette occasion, il découvre Moses Finley qui avait publié en 1954 The World of Odysseus. "Quand je lus Finley, à l'occasion d'un gros article que je publiai en 1963 dans les Annales, j'eus le sentiment, largement justifié, que j'avais découvert un historien. Pas une ligne de Finley n'avait alors été traduite en français ${ }^{42}$. " La lecture de l'article montre toutefois que Vidal-Naquet n'hésite pas à exprimer des réserves sur l'analyse finleyienne des poèmes homériques ${ }^{43}$. L'essentiel était sans doute que Finley représentait l'école historique contre un Companion qu'il critiquait et qu'il lisait comme "le manifeste des homérisants archéologues ${ }^{44}$ ". L'année suivante, en 1964, à Paris, il rencontre Finley qui bientôt le fait inviter à la Troisième conférence d'histoire économique qui se tint à Munich en 1965, sous la présidence de Fernand Braudel. Au cours de cette même année, il publie une préface critique à l'ouvrage de Karl August Wittfogel, Le Despotisme oriental. Marx considérait qu'il existait un mode de production asiatique ayant pour principale caractéristique l'immobilisme et le despotisme ; Wittfogel pensait que le stalinisme en constituait une illustration. Ancien communiste, l'homme était aussi celui qui avait attaqué l'auteur du Monde d'Ulysse et qui était responsable de son départ de Rutgers vers l'Angleterre et Cambridge ${ }^{45}$.

Nul doute que Vidal-Naquet trouvait dans la situation de Finley des échos avec sa propre situation consécutive à ses engagements au cours de la guerre d'Algérie. Emporté par le tourbillon algérien et l'affaire Maurice Audin, il avait fait preuve de courage

40 Outre P. Vidal-Naquet, Mémoires. 2. Le trouble et la lumière (1955-1998), Paris, 1998, cf. Cl. Mossé, « Rencontre avec M.I. Finley : l'histoire économique et sociale dans l'œuvre de Pierre Vidal-Naquet ", in Fr. Hartog et al., Pierre Vidal-Naquet, un historien dans la cité, Paris, 2007, p. 110-122.

41 Cf. Vidal-Naquet, Mémoires, p. 162.

42 Ibid., p. 171. Cf. ID, "Homère et le monde mycénien, à propos d'un livre récent et d'une polémique ancienne ", AESC 18 (1963), p. 703-719.

43 "Homère ", p. 716, n. 6. Il abandonne ses réserves dans son "Économie et société dans la Grèce ancienne : l'œuvre de Moses I. Finley ", in ID., La démocratie grecque vue d'ailleurs, Paris, 1990, p. 57-64 (= Archives européennes de sociologie 6, 1965).

44 Ibid., p. 712.

45 Pierre Vidal-Naquet le mentionne dans ses Mémoires, sans qu'il soit possible d'établir une relation de cause à effet entre l'amitié naissante entre les deux hommes et cette préface. 
politique et physique, tout en subissant les conséquences de ses engagements. Dans ses Mémoires, il rapporte n'avoir dû son détachement auprès du CNRS qu'aux interventions conjointes d'André Aymard et d'Henri-Irénée Marrou. Il n'est donc pas surprenant que, dès le début de son article "Économie et société dans la Grèce ancienne : l'œuvre de Moses I. Finley » paru en 1965, il rappelle que l'historien américain a dû quitter son pays d'origine en raison du maccarthysme ${ }^{46}$. Mais il serait erroné de s'en tenir à cette lecture politique. C'est bien en raison de conceptions partagées que les deux hommes s'apprécièrent et nouèrent une relation amicale. Pour le dire en empruntant les mots à la sociologie de Pierre Bourdieu, homologie structurale et affinités d'habitus marchent ici main dans la main. Les trois points abordés par Vidal-Naquet dans son article - le monde homérique, l'esclavage et les liens entre économique et politique - constituent autant d'occasions de faire apparaître la proximité existant entre eux. C'est dans la troisième partie que cette conception commune se voit le mieux. Elle s'entend avec force dans cette exhortation : "Fuyons donc les anachronismes, mais surtout munissons-nous d'un vocabulaire, d'un appareil conceptuel adapté à l'objet de l'étude ${ }^{47}$. "Vidal reprend alors les conclusions du rapport finleyien prononcé lors de la Deuxième Conférence d'histoire économique d'Aix, dans lequel il lit une invitation à « désembourber l'histoire économique de l'Antiquité des marécages et des formules toutes faites dans lesquelles elle se complaît : réexamen de la différenciation entre marchandises de luxe et marchandises de première nécessité ; étude systématique des relations avec le monde barbare [...] ; analyse différentielle du monde grec lui-même ». Il retient également la nécessaire constitution d'un " corpus complet des règles et des procédures des marchés 48 ".

Néanmoins, cette indéniable proximité ne doit pas obérer le fait que cet article n'est pas seulement une présentation en français des principales thèses finleyiennes. Il faut souligner avec Claude Mossé que ce texte « ouvrait [...] de nouvelles directions de recherche, en même temps qu'il donnait au débat sur l'économie grecque antique une orientation originale ", montrant par là même qu'il est sans doute délicat de parler de réception de l'œuvre de Finley, sauf à aborder dans le même temps la réception de cette tradition française dans l'œuvre finleyienne ${ }^{49}$. De ce point de vue, la parution en 1972 d'Économies et sociétés en Grèce ancienne, écrit avec Michel Austin, élève de Finley, doit être appréciée comme une contribution à la rédaction de The Ancient Economy, publié l'année suivante. La collection U2 imposait un format universitaire, sur le modèle du cours et des travaux dirigés, en l'occurrence une présentation générale et des textes ${ }^{50}$. Mais l'ouvrage est assurément à lire comme une prise de position historiographique.

46 Au sujet de cet article, il confie dans ses Mémoires : "Naturellement, je l'avais montré à Finley dont j'avais fait la connaissance à Paris, en 1964 " (Mémoires, p. 172).

47 "Économie et société dans la Grèce ancienne ", p. 76.

48 Ibid., p. 80.

49 Cl. Mossé, «Rencontre ", p. 119.

50 Selon Claude Mossé, il faut lire la première partie comme, au moins partiellement, un travail en commun, même si l'éditeur en assigne la seule paternité à Austin (ibid., p. 120). 
L'influence de Moses Finley est patente et explicite : "Nous remercions aussi M.I. Finley qui, plus que tout autre historien contemporain, a renouvelé notre perception de l'histoire économique et sociale du monde grec ", écrivent les auteurs dans leur préface $^{51}$. Elle se lit du reste dans la référence à Max Weber, manquante dans l'article de 1954 d'Édouard Will, et à Karl Polanyi. Mais les auteurs remercient aussi Sally Humphreys, auteure en 1969 d'un long article consacré à l'œuvre polanyienne ${ }^{52}$. Ils reprennent surtout la notion d'encastrement, " c'est-à-dire l'impossibilité d'étudier l'économie grecque isolément et en faisant abstraction des cadres social et institutionnel de l'histoire grecque ${ }^{53}$ ". Conformément au modèle polanyien, les auteurs consacrent une partie de leur ouvrage aux valeurs de la société grecque qui expliquent le mépris pour l'artisan et en même temps pourquoi ce dernier est " le héros secret de l'histoire grecque $^{54}$ ". Ces profondes similitudes entre Économies et sociétés en Grèce ancienne et The Ancient Economy peuvent être expliquées par quatre éléments : la place marginale de la sociologie par rapport à l'ethnologie/anthropologie ; le postulat de l'altérité des anciens Grecs ; l'aventure de la psychologie historique autour de Jean-Pierre Vernant ; et, soubassement décisif, la nature du champ disciplinaire.

Il est en effet paradoxal de constater que si Finley récusait le recours à l'anthropologie au-delà de l'époque archaïque et s'inspirait fortement de Max Weber, Pierre Vidal-Naquet est au contraire fortement influencé par le structuralisme, même s'il n'ignore pas la sociologie webérienne. Cette situation parait être une singularité française ${ }^{55}$. Alors que le débat avait été vif à la fin du XIX et au début du XX $\mathrm{XX}^{\mathrm{e}}$ siècle entre l'histoire et la sociologie, après 1945, la discussion semble être polarisée autour de l'ethnologie et de l'histoire. Mais il avait fallu pour cela mettre hors jeu la sociologie. Claude Lévi-Strauss s'en était chargé dès 1949 dans la Revue de métaphysique et de morale ${ }^{56}$. Il y écrivait notamment : "Le terme sociologie [...] n'a pas encore réussi à mériter, depuis le début de ce siècle, le sens général de corpus de l'ensemble des sciences sociales qu'avaient rêvé pour lui Durkheim et Simiand. " "Philosophie sociale " en France et dans de nombreux pays européens, «si on voit en elle, comme c'est le cas dans les pays

51 M. Austin \& P. Vidal-Naquet, Économies et sociétés en Grèce ancienne, Paris, [2007], p.V. Cf. ici même P. Ismard, p. 39-53 (que nous remercions pour la communication de son texte).

52 Article repris dans S. Humphreys, Anthropology and the Greeks, Londres, 1978, p. 31-73.

53 Ibid., p. 19. Le mot est aujourd'hui consacré. Austin et Vidal-Naquet rendent l'embeddedness polanyien par le mot français intégration.

54 Encyclopadia universalis (t. VII), Paris, 1970, repris dans P. VIDAL-NAQUET, Le chasseur noir, Paris, 1991, en particulier p. 31-33.

55 Cf. toutefois le compte rendu (1956) imprégné d'une forte tonalité sociologique de The World of Odysseus par L. Gernet (repris dans Les Grecs sans miracle, p. 138-141).

56 Cl. LÉvi-Strauss, "Histoire et ethnologie ", Revue de métaphysique et de morale 54 (1949), p. 363-391 avec Fr. Hartog, "Le regard éloigné : Lévi-Strauss et l'histoire ", in ID., Évidence de l'histoire. Ce que voient les historiens, Paris, 2005, p. 216-233. 
anglo-saxons, un ensemble de recherches positives portant sur l'organisation et le fonctionnement des sociétés du type le plus complexe, la sociologie devient une spécialité de l'ethnographie, sans pouvoir prétendre, en raison même de la complexité de son objet, à des résultats aussi précis et valables que celle-ci, dont la considération offre ainsi, du point de vue de la méthode, une plus grande valeur topique ${ }^{57}$ ". La véritable discussion ne pouvait donc avoir lieu qu'entre l'histoire et l'anthropologie, forme anoblie de l'ethnologie, Claude Lévi-Strauss ayant substitué « à la désignation traditionnelle de la discipline l'appellation anglaise d'anthropologie et en cumulant ainsi les prestiges du sens allemand [...] et la modernité du sens anglo-saxon ${ }^{58}$ ". François Hartog a bien décrit les différentes étapes de ce débat ${ }^{59}$. Il semble que pour les historiens de l'Antiquité, le structuralisme et plus généralement l'anthropologie apparaissent comme une pratique de l'estrangement ${ }^{60}$. Desperately foreign, les anciens Grecs semblaient se prêter naturellement aux parallèles anthropologiques ${ }^{61}$.

Certes, il serait erroné de faire de Pierre Vidal-Naquet un structuraliste, qualificatif qu'il ne fait du reste pas sien. Avec modestie, toujours dans ses Mémoires, au moment de se définir, il se lance dans l'énumération de ce qu'il n'est pas, ni linguiste, ni philologue, ni archéologue, ni épigraphiste, ni papyrologue, ni psychologue, ni sociologue, tout en indiquant les compétences acquises dans ces différents domaines par sa fréquentation auprès des meilleurs représentants de ces savoirs. "Je ne suis pas un helléniste dans toute la force du terme à la façon de Jean Pouilloux, capable à la fois de commenter une inscription ou une tragédie et d'animer un chantier de fouilles. [...] Un esprit ironique dirait peut-être que je suis un "polymorphe souple". [...] Il est vrai que, toute ma vie, je me suis voulu généraliste. [...] Cela dit, j’ai creusé de mon mieux mon sillon grec comme le recommandait Marrou à ses élèves, mais je n'ai jamais cessé de regarder ailleurs, parfois du côté de Rome ou de Jérusalem, parfois du côté du Moyen Âge, parfois dans l'époque moderne et contemporaine ${ }^{62}$. "Agrégé d'histoire, il ne découvre Claude Lévi-Strauss qu'en 1965, lisant successivement au cours de l'été Tristes Tropiques, Mythologiques 1 et Le cru et le cuit. Sans doute l'influence de Vernant est-elle en partie à l'origine de ces lectures, puisque ce dernier avait publié en 1963, dans L'Homme, un article sur le couple Hestia-Hermès. Le chasseur noir en est le fruit. À ce sujet, il précise « que l'objet de [sa] recherche n'est pas essentiellement le mythe comme le croient beaucoup ; il se situe au croisement de la pensée et de la société telles que peut les comprendre et les analyser un historien ${ }^{63}$ ». Toutefois, en raison même du domaine choisi, l'étude des représentations

57 Cl. Lévi-Strauss, « Histoire et ethnologie », p. 364.

58 P. Bourdieu, Esquisse, p. 57.

59 Fr. HARTOG, «Le regard éloigné ».

60 Ibid., p. 232.

61 L'expression deseperatly foreign est empruntée à l'introduction du livre de M.I. FinLEY, Aspects of Antiquity (Londres, 1960), repris dans On a perdu la guerre de Troie, Paris, 1989, p. 7-12.

62 P. Vidal-Naquet, Économies et sociétés, p. 216-217.

63 Ibid., p. 229. 
et non l'établissement des faits, selon ses propres mots, il ne pouvait que marquer un vif intérêt pour une vaste fresque comme The Ancient Economy ${ }^{64}$.

L'influence et l'amitié de Jean-Pierre Vernant ont assurément joué un rôle tout aussi important. Pierre Vidal-Naquet le rencontre à l'automne 1960 et assiste à ses séminaires à la VI ${ }^{\mathrm{e}}$ section de l'EPHE. C'est à ce moment que Vernant fixe son programme de psychologie historique : "Nous recherchons ce qu'a été l'homme lui-même, cet homme grec ancien que l'on ne peut séparer du cadre social et intellectuel dont il est à la fois le créateur et le produit ${ }^{65}$. " Cette démarche s'incarnait dans un mot d'ordre, emprunté à Z. Barbu, dans ses Problems of Historical Psychology, "Back to the Greeks ${ }^{66}$ ! » De telles considérations détonnaient dans le champ disciplinaire des années 1960 dont la description faite par Vidal-Naquet donne une idée assez précise. «Il m'est arrivé de dire que les études grecques, en France, à la fin des années 1960, étaient marquées par la domination d'une Église, la Sorbonne, à laquelle faisaient face plusieurs sectes qui résistaient de leur mieux : secte marxiste à Besançon avec, à sa tête, Pierre Lévêque, secte philologique à Lille, avec Jean Bollack et ses disciples, secte anthropologique à l'École avec Jean-Pierre Vernant et ses amis ${ }^{67}$. " Les prises de position sont à comprendre par rapport à la position dominante de la Sorbonne et plus généralement de la philologie : "Toute une partie de mes travaux, principalement pendant mes débuts, a été marquée par la volonté de prouver aux autres et de me prouver à moi-même que j'étais capable, sur le plan de l'érudition, de faire aussi bien que n'importe quel helléniste classique, de la Sorbonne ou d'ailleurs ${ }^{68}$. "Sans nier la rupture dans l'histoire antique que représentait la Grèce ancienne, celle-ci ne résultait pas de la langue grecque comme le voulaient les philologues de la Sorbonne, mais des institutions, de la polis, de cet espace du politique décrit dans Clisthène l'Athénien et qui tranchait avec le monde, assurément grec par la langue, des tablettes mycéniennes. Vernant, Vidal et Finley décrivaient donc une même histoire.

L'économie antique de Finley n'est pourtant pas éditée dans la collection «Textes à l'appui ", collection dirigée par Pierre Vidal-Naquet aux éditions Maspéro, qui refusent le manuscrit, et qui avait été créée peu avant, au moment de la publication d'un recueil d'articles de Jean-Pierre Vernant, Mythe et pensée chez les Grecs. C'est dans la collection Le sens commun, aux éditions de Minuit, créée en 1965 également, que la traduction est publiée. Il n'est pas possible de dire si la relation que Pierre Vidal-Naquet avait contractée avec le directeur de celle-ci, Pierre Bourdieu, lors de leur séjour commun à Lille a joué. Il est certain néanmoins qu'un livre affirmant avec tant de force l'encastrement de l'économique dans le social, ce qui correspond à la société indifférenciée des sociologues

64 Ibid., p. 369.

65 J.-P. Vernant, Entre mythe et politique, Paris, 1996, p. 9.

66 Ibid., p. 10.

67 P. Vidal-NaQUeT, Économies et sociétés, p. 209.

68 Ibid., p. 218. 
durkheimiens, point d'origine de la division du travail social, ne pouvait que séduire le directeur du Sens commun, collection qui accueillait prioritairement des ouvrages de philosophie et de sociologie, et qui faisait au même moment une place d'honneur à Émile Durkheim, en publiant les trois volumineux tomes de ses Écrits. Pour Pierre Bourdieu donc, la traduction française de L'économie antique contribuait à son projet global d'unification de la science sociale auquel il associe son entreprise éditoriale ${ }^{69}$.

À l'évidence, l'ouvrage ne fut pas reçu en France dans cette perspective. Les historiens de l'économie, en particulier bordelais, y ont très vite lu une prise de position primitiviste, choisissant de fait l'angle économique pour rendre compte de la thèse finleyienne, c'est-à-dire pour prendre position. Dès lors, la notion de marché et plus généralement la question des échanges prenaient une importance capitale. D'autres historiens, comme Pierre Vidal-Naquet, y lurent avant tout une mise en évidence salutaire de l'altérité grecque, négligeant la dimension proprement sociologique revendiquée par Finley, au profit du regard anthropologique, du retour aux Grecs qu'avait annoncé Jean-Pierre Vernant, rompant ainsi avec la vision édifiante alors en vogue à la Sorbonne. Si l'Économie antique se prête à des lectures diverses, c'est naturellement parce que ces différents éléments s'y trouvent. Mais, les conditions d'un progrès dans l'écriture de l'histoire supposent de ne pas prolonger à l'infini des perspectives ouvertes à partir de positions qui structuraient le champ disciplinaire à un moment donné ${ }^{70}$. Elle requiert - après avoir défini l'espace des possibles intellectuels dans lequel la réception d'une œuvre, en l'occurrence celle de Finley, s'inscrit - d'envisager les conditions sinon de son dépassement, du moins de son élargissement, en fonction d'autres enjeux ${ }^{71}$. Aujourd'hui, les reconfigurations à l'intérieur des sciences sociales et les interrogations sur la place que l'économie pourrait y occuper constituent un puissant moteur pour une relecture française de l'Économie antique.

Christophe PÉBARTHE

Université de Bordeaux 3

UMR 5607 Ausonius

Maison de l'Archéologie

8, esplanade des Antilles

33607 Pessac Cedex

christophe.pebarthe@u-bordeaux3.fr

69 P. Bourdieu, Science de la science et réflexivité, Paris, 2001, p. 197.

70 Nous ne pouvons que renvoyer ici à l'article de 1977 de M.I. FinLEY, "Progrès dans l'écriture de l'histoire ", in EAD, Mythe, mémoire, histoire, Paris, 1981, p. 143-173.

71 Chr. Charle, Homo Historicus. Réflexions sur l'histoire, les historiens et les sciences sociales, Paris, 2013, p. 16 parle de "gymnastique pour élaborer les bonnes questions pour aujourd'hui, non pour gagner des victoires faciles dans des combats déjà livrés où l'on soutient, bien entendu, les vainqueurs désignés par la postérité ». 